\title{
CONHECIMENTO SOBRE A VERIFICAÇÃO DE PRESSÃO ARTERIAL DOS ENFERMEIROS DE UM HOSPITAL ESCOLA DO INTERIOR PAULISTA
}

\author{
Ariane Campos Gervazoni, Kellen Santos Obregon Lopes, Milena Colonhese Camargo \\ Universidade do Oeste Paulista - UNOESTE, Curso de Enfermagem, Presidente Prudente, SP. E-mail: \\ milenacolonhese@uol.com.br
}

\begin{abstract}
RESUMO
A equipe de enfermagem é responsável pela maior parte da aferição da pressão arterial, sendo um procedimento rotineiro e de grande complexidade teórico-pratica e se determinados princípios para sua realização não forem atendidos estará sujeitos a erros. Considerando o procedimento de extrema importância para diagnóstico e tratamento, objetivou-se identificar o conhecimento teórico dos enfermeiros de um hospital de ensino sobre a técnica de verificação da pressão arterial. Trata-se de uma pesquisa descritiva, transversal, prospectiva, tipo estudo de caso com abordagem quantitativa. Foi elaborado um instrumento com 15 itens relacionados ao preparo do paciente e sobre a técnica propriamente de acordo com as VI Diretrizes Brasileiras de Hipertensão. Verificou-se que os 68 enfermeiros que aceitaram participar do estudo, demonstraram conhecimento satisfatório em relação ao preparo do paciente para a verificação da pressão arterial. Sobre os itens relacionados à técnica das 12 questões analisadas, somente cinco apresentaram um total de acertos superior a $80 \%$. Conclui-se que os enfermeiros apresentaram sérias deficiências em alguns conceitos teóricos, essenciais na técnica de verificação da pressão arterial, reforçando a necessidade de um aprofundamento desse assunto nos cursos de graduação, bem como treinamentos aos referidos profissionais baseados nas recomendações das Diretrizes Brasileiras de Hipertensão Arterial. Sendo assim será apresentada pelas pesquisadoras à gerente de enfermagem da instituição participante uma proposta para implantação de um protocolo de verificação da pressão arterial. Palavras-chave: enfermagem, conhecimento, pressão arterial.
\end{abstract}

\section{KNOWLEDGE ABOUT THE CHECKING OF BLOOD PRESSURE OF NURSES OF A SCHOOL HOSPITAL OF THE PAULISTA INTERIOR}

\begin{abstract}
The nursing staff is responsible for most of the blood pressure measurement, and a routine procedure and complexity theory-practice and if certain principles for its realization are not attended will be subject to errors. Considering the procedure of utmost importance for diagnosis and treatment, it aimed to identify the theoretical knowledge of nurses on the technique of indirect measurement of blood pressure. This is a descriptive, cross-sectional, prospective, case study with a quantitative approach. To conduct this study is a questionnaire addressing theoretical aspects related to knowledge about the technique of blood pressure measurement to nurses at a general teaching hospital in São Paulo state after authorization and consent of the subjects who agree to participate by signing the Informed Consent and Informed. The instrument consists of 15 items, containing response options: true, false and do not know and will be collected immediately after its completion. The data collected will be analyzed using means and percentages presented in tables.
\end{abstract}

Keywords: Keywords: nursing knowledge, blood pressure.

\section{INTRODUÇÃO}

A Pressão Arterial (PA) é definida como a força exercida pelo sangue na parede das artérias, tradicionalmente referida em milímetro de mercúrio (mmHg). É um parâmetro fisiológico indispensável durante uma investigação diagnóstica de hipertensão arterial ${ }^{1,2}$. 
De acordo com Boll et al. ${ }^{3}$ a Hipertensão Arterial afeta um terço da população mundial, sendo considerado o maior fator de risco para causar insuficiência cardíaca, doença coronariana, falência renal. A probabilidade de um individua ao longo de sua vida tornar-se hipertenso é de $90 \%$, sendo um grande desafio para a saúde publica no Brasil. Calcula-se que $30 \%$ da população com mais de 40 anos venha adquirir pressão arterial elevada. Com base nesses estudos é de suma importância que os profissionais da saúde tenham conhecimentos epidemiológicos, diagnósticos e terapêuticos da Hipertensão Arterial Sistêmica (HAS).

Brito et al. $^{4}$ descreveram que a classificação da HAS sofreu muitas mudanças nas ultimas décadas, considerando hoje hipertensão arterial para indivíduos adultos (com 18 anos ou mais) a pressão sistólica persistente maior ou igual a $140 \mathrm{mmHg}$, e pressão diastólica persistente maior ou igual a $90 \mathrm{mmHg}$, em pessoas que não fazem uso de medicamentos anti-hipertensivo.

Segundo Didier e Guimarães ${ }^{5}$ esse problema tem preocupado o Ministério da Saúde, pelos aspectos humanos e econômicos. As doenças cardiovasculares são responsáveis por $14 \%$ das internações (representando 16,2\% do orçamento da saúde), aposentadoria precoce, sendo considerada a primeira causa de morte no Brasil.

A medida da pressão arterial (PA) é o procedimento usado no diagnóstico da HAS para o acompanhamento e avaliação da eficácia do tratamento dos pacientes hipertensos. Este procedimento é de extrema importância e merece atenção dos profissionais da saúde, que devem ser orientados e treinados sobre a forma correta de execução, pois a falta de conhecimento interfere a assistência prestada. É um procedimento rotineiro e, se determinados princípios para sua realização não forem atendidos, estará sujeito a vários erros. A medida incorreta pode considerar um individuo hipertenso como normotenso, privando-o do tratamento ou diagnosticar a hipertensão em normotensos, expondo-os ao tratamento desnecessário ${ }^{1,6,7}$.

Para verificar a pressão arterial pode ser utilizado o método direto que fornece a pressão direta ou intra-arterial exigindo equipamentos mais sofisticados, por ser um método invasivo. 0 método indireto é mais simples e utiliza a técnica auscultatória com estetoscópio e um esfigmomanometro constituído por uma braçadeira inflável que envolve o braço, ligado a um manômetro, sistema de válvulas, pera e tubos de borracha ${ }^{2}$.

Durante o processo de verificação da pressão arterial devem-se levar em conta vários cuidados básicos em relação ao paciente, ao equipamento, à técnica de aferição, ao correto registro dos valores e também à interpretação dos dados ${ }^{6,8}$.

Estudos mostram que há necessidade de padronização dos critérios utilizados na realização deste procedimento, ou seja, deve ser feita de forma sistematizada, pois os valores reais determinam à conduta e o tratamento adequado para a doença quando existente. É fundamental a diminuição de erros na medida da pressão arterial, sendo necessários aparelhos calibrados, dimensões adequadas de manguito, conservação e manutenção das peras e capacitação dos profissionais de enfermagem ${ }^{9-11}$.

Veiga et al. ${ }^{9}$ reforçam que na pratica assistencial entre diferentes profissionais, e em diversos locais, a medida da pressão arterial não é realizada de forma correta, podendo comprometer valores obtidos e, consequentemente, o diagnóstico, tratamento e controle da hipertensão.

O passo inicial para a realização da técnica é o preparo adequado do paciente, que deverá estar calmo e descansado em ambiente confortável, informado sobre o procedimento que será realizado. $O$ equipamento a ser utilizado deverá estar devidamente calibrado a menos de seis meses, e o manguito de tamanho adequado, onde sua largura deve corresponder a $40 \%$ da circunferência braquial e o seu comprimento de 80 a $100 \%$ do tamanho do braço. O examinador também deverá estar posicionado de forma confortável e os valores obtidos devem ser anotados logo após evitando possível esquecimento ${ }^{8,11,12}$.

A equipe de enfermagem é responsável pela maior parte das aferições de pressão arterial, tanto nas Unidades Básicas de Saúde quanto nos hospitais, portanto a verificação da Pressão Arterial de maneira precisa deve ser de interesse tanto dos órgãos formadores quanto dos setores de educação continuada. A presença do enfermeiro junto aos pacientes hipertensos é de grande importância, devido ao seu papel como educador motivando os pacientes à adesão ao tratamento e capacitando os demais profissionais da equipe de enfermagem nas 
atividades que são de sua competência, desenvolvendo intervenções seguras baseadas em evidencias cientificas ${ }^{13}$.

De acordo com as VI Diretrizes Brasileiras de Hipertensão Arterial o papel e ações específicas são estabelecidas para cada participante da equipe multiprofissional, onde técnicos e auxiliares de enfermagem estão subordinados à supervisão do enfermeiro, portanto, esses necessitam estar devidamente preparados e conscientizados sobre a importância da atuação junto aos pacientes com $\mathrm{HAS}^{13}$.

Levando em consideração que grande número de profissionais da saúde apresenta dúvidas nesse assunto, o presente estudo teve como objetivo identificar o conhecimento teórico de enfermeiros de um hospital de ensino sobre a técnica de verificação da pressão arterial de acordo com a recomendação da VI Diretrizes Brasileiras de Hipertensão Arterial e posteriormente propor para a instituição a implantação de um protocolo de verificação da pressão arterial.

\section{METODOLOGIA}

Trata-se de uma pesquisa descritiva, transversal, prospectiva, tipo estudo de caso com abordagem quantitativa realizado no período de agosto a outubro de 2013.

Segundo Polit et al. $^{14}$ a pesquisa quantitativa reúne informações numéricas que resultam de mensuração formal, sendo analisada com procedimentos estatísticos.

Esta pesquisa será do tipo estudo de caso, pois de acordo com Severino ${ }^{15}$ é um tipo de pesquisa que delimita um caso em particular que representa um conjunto de casos análogos devendo ser significativo e bem representativo.

O projeto foi protocolado e aprovado pelo Comitê de Ética em Pesquisa da Unoeste, sob o protocolo núm. 1594.

Os sujeitos desta pesquisa foram 68 enfermeiros de um hospital de ensino do interior paulista. Segundo informações da gerente de enfermagem do hospital, o quadro de enfermeiros é composto por 136 profissionais distribuídos em 31 setores.

O instrumento para identificação do conhecimento sobre a técnica de verificação da pressão arterial foi elaborado com 15 itens relacionadas ao preparo do paciente e sobre a técnica propriamente, contendo opções de resposta: verdadeiro, falso e não sei, elaborado pelos pesquisadores de acordo com a tabela de recomendações para a medida da pressão arterial das VI Diretrizes Brasileiras de Hipertensão ${ }^{16}$.

O conhecimento foi considerado satisfatório com o nível de acertos acima de $80 \%$, por se tratar de um hospital de ensino.

O procedimento de coleta e análise de dados seguiu as seguintes etapas:

1. Apresentação da proposta e solicitação de autorização à Direção da Instituição escolhida;

2. Explicação sobre as contribuições sociais e acadêmicas da pesquisa, durabilidade e os passos a serem percorridos;

3. Autorização e consentimento dos sujeitos que aceitarem participar através da assinatura do Termo de Consentimento Livre e Esclarecido;

4. O instrumento foi aplicado pessoalmente pelos pesquisadores, em data previamente agendada, a 68 enfermeiros que aceitaram participar da pesquisa no local de trabalho antes do turno, ao final ou em período de intervalo, sendo os períodos diurno, vespertino e noturno em uma sala reservada em cada unidade, sem tempo determinado para responder o instrumento. Foi entregue ao profissional junto com o instrumento um envelope e uma etiqueta para lacrar, garantindo assim o anonimato. Foram excluídos da pesquisa os enfermeiros de férias, os afastados, e os que não aceitaram participar do estudo.

5. Os pesquisadores recolheram o envelope lacrado com o instrumento logo após seu preenchimento.

6. Os dados coletados foram analisados através de porcentagem simples e apresentados em tabelas.

\section{RESULTADOS}

Do quadro de enfermeiros convidados, 68 aceitaram participar da pesquisa. Analisando a Tabela 1, verifica-se que o tempo de formação profissional e o tempo de trabalho na instituição predominou entre 1 e 10 anos, $10(14,7 \%)$ dos participantes responderam ter recebido treinamento sobre aferição da pressão arterial. 
Tabela 1. Características sociodemográficas dos participantes da pesquisa.

\begin{tabular}{lcc}
\hline Características & $\begin{array}{c}\text { Enfermeiros } \\
(\mathrm{n}=68)\end{array}$ \\
\hline Tempo de formação & $\mathrm{F}$ & $\%$ \\
profissional (anos) & 39 & 57,3 \\
$1-10$ & 6 & 8,82 \\
$11-20$ & 1 & 1,47 \\
$21-30$ & 22 & 32,35 \\
Não respondeu & 68 & 100 \\
Total & $\mathrm{F}$ & $\%$ \\
Tempo de instituição (anos) & 10 & 14,70 \\
$<1$ & 30 & 44,11 \\
$1-10$ & 9 & 13,23 \\
$11-20$ & 0 & 0 \\
$21-30$ & 19 & 27,94 \\
Não respondeu & 68 & 100 \\
Total & $\mathrm{F}$ & $\%$ \\
Recebeu treinamento sobre & & \\
aferição da pressão arterial & 10 & 14,70 \\
Sim & 47 & 69,11 \\
Não & 11 & 16,17 \\
Não respondeu & 68 & 100 \\
Total &
\end{tabular}

Em relação ao preparo do paciente para a verificação da PA, foi evidenciado que mais de $80 \%$ dos participantes da pesquisa acertaram as respostas, considerado o resultado satisfatório para um hospital escola (Tabela 2).

Tabela 2. Distribuição das respostas dos participantes da pesquisa de acordo com o preparo do paciente para a verificação da pressão arterial.

\begin{tabular}{|c|c|c|c|c|}
\hline \multirow[b]{2}{*}{ Itens relacionados ao preparo do paciente } & \multicolumn{3}{|c|}{ Distribuição respostas $(\mathrm{n}=68)$} & \multirow[b]{2}{*}{ Total } \\
\hline & $\begin{array}{c}\text { Corretas } \\
\text { n (\%) }\end{array}$ & $\begin{array}{c}\text { Incorretas } \\
\text { n (\%) }\end{array}$ & $\begin{array}{c}\text { Não sei } \\
\text { n (\%) }\end{array}$ & \\
\hline $\begin{array}{l}\text { 1- Explicar o procedimento deixá-lo em repouso por } 5 \\
\text { minutos, instruindo-o a não conversar durante o } \\
\text { procedimento. }\end{array}$ & $66(97)$ & $2(3)$ & $0(0)$ & 68 \\
\hline $\begin{array}{l}\text { 2- Certificar-se de que não está com a bexiga cheia, } \\
\text { praticou exercícios físicos, ingeriu bebidas alcoólicas, café } \\
\text { ou alimentos e fumou. }\end{array}$ & $59(87)$ & $8(12)$ & $1(1)$ & 68 \\
\hline $\begin{array}{l}\text { 3- Posição sentada, pernas descruzadas, pés apoiados no } \\
\text { chão, dorso recostado na cadeira e relaxado. O braço deve } \\
\text { estar na altura do coração, livre de roupas, apoiado, com a } \\
\text { palma da mão voltada para cima e o cotovelo ligeiramente } \\
\text { fletido. }\end{array}$ & $59(87)$ & $7(10)$ & $2(3)$ & 68 \\
\hline
\end{tabular}

Sobre os itens relacionados à técnica da pressão arterial, das 12 questões analisadas, somente cinco apresentaram um total de acertos superior a $80 \%$, sendo o item sobre a localização do manguito sobre a artéria braquial com maior porcentagem de acerto (88\%). Os demais itens 
atingiram uma média de 60,5\% de acertos, o item com menor porcentagem (22\%) corresponde ao conhecimento sobre se os batimentos

Tabela 3. Distribuição das respostas dos participantes da pesquisa, de acordo com a técnica da aferição da pressão arterial.

Itens relacionados ao preparo do paciente
braço do cliente, para selecionar o manguito de tamanho adequado ao braço.

5- Colocar o manguito, sem deixar folgas, 2 a $3 \mathrm{~cm}$ acima da fossa cubital, centralizando o meio da parte compressiva do manguito sobre a artéria braquial.

6- Estimar o nível da pressão sistólica pela palpação do pulso radial.

7- Palpar a artéria braquial na fossa cubital e colocar a campânula ou o diafragma do estetoscópio sem compressão.

8- Inflar rapidamente até ultrapassar 20 a 30 mmHg o nível estimado da pressão sistólica, e proceder à deflação lentamente $2 \mathrm{mmHg} / \mathrm{s}$

9- Determinar a pressão sistólica pela ausculta do primeiro som (fase I de Korotkoff) e a pressão diastólica no desaparecimento dos sons (fase $\mathrm{V}$ de Korotkoff).

10- Se os batimentos persistirem até o nível zero, determinar a pressão diastólica no abafamento dos sons (fase IV de Korotkoff) e anotar valores da sistólicodiastólica e zero.

11- Se houver dúvidas nos valores, sugere-se esperar em torno de um minuto para nova medida.

12- Devemos informar os valores da pressão arterial obtidos para o cliente.

13- Sempre anotar os valores exatos sem "arredondamentos" e o braço em que a pressão arterial foi aferida.

14- Para pacientes obesos são necessários manguitos mais longos e largos, em braços com circunferência superior a $50 \mathrm{~cm}$.

15- Nas gestantes a pressão arterial deve ser aferida com os mesmos equipamentos e com a mesma técnica recomendada para adultos. persistirem até o nível zero, sendo considerado conhecimento insatisfatório (Tabela 3).

\begin{tabular}{cccc}
\multicolumn{4}{c}{ Distribuição respostas (n=68) } \\
$\begin{array}{c}\text { Corretas } \\
\mathbf{n}(\%)\end{array}$ & $\begin{array}{c}\text { Incorretas } \\
\mathbf{n}(\%)\end{array}$ & $\begin{array}{c}\text { Não sei } \\
\mathbf{n}(\%)\end{array}$ & Total \\
$49(72)$ & $12(18)$ & $7(10)$ & 68 \\
$61(90)$ & $7(10)$ & $0(0)$ & 68 \\
$38(56)$ & $23(34)$ & $7(10)$ & 68 \\
$60(88)$ & $7(10)$ & $1(1)$ & 68 \\
& & & \\
$40(59)$ & $19(28)$ & $9(13)$ & 68
\end{tabular}

$51(75) \quad 7(10) \quad 10(15) \quad 68$

$15(22) \quad 15(22) \quad 38(56) \quad 68$

$50(73) \quad 15(22) \quad 3(4) \quad 68$

$57(84) \quad 7(10) \quad 4(6) \quad 68$

$60(88) \quad 7(10) \quad 1(1) \quad 68$

$45(66) \quad 11(16) \quad 12(18) \quad 68$

$60(88) \quad 6(9) \quad 2(3) \quad 68$ 


\section{DISCUSSÃO}

Este estudo foi realizado com a intenção de avaliar o conhecimento teórico da medida da pressão arterial dos enfermeiros de um hospital de ensino do interior paulista, segundo as recomendações da VI Diretrizes de Brasileira de Hipertensão ${ }^{16}$ e propor para a instituição a implantação de um protocolo de verificação. Outros pesquisadores também vêm demonstrando grande preocupação em identificar se os profissionais da saúde apresentam conhecimento teórico e prático satisfatório sobre o procedimento, uma vez que está ligado diretamente com a avaliação de um parâmetro vital.

Em relação às características dos participantes da pesquisa, foi observado que o tempo de formação profissional e o tempo de trabalho na instituição predominou entre 1 e 10 anos. Resultados semelhantes foram descrito no estudo de Lima e Gusmão7, avaliando o conhecimento teórico e prático de auxiliares de enfermagem sobre medida da pressão arterial, em que a maioria da população do estudo tinha tempo de formação inferior a 10 anos, o que leva a supor que o conhecimento teórico adquirido durante a formação é recente contribuindo para maiores índice de acerto o que não ocorreu no presente estudo. Veiga et al. ${ }^{9}$ avaliando a técnica da medida da pressão arterial pelos profissionais de saúde, também observou o tempo de formação entre 1 a 5 anos e com resultados insatisfatórios, podendo levar a entender que os cursos de graduação podem não estar preparando adequadamente os profissionais para esse procedimento específico.

Quanto ao preparo do paciente para a realização da medida os enfermeiros participantes do presente estudo apresentaram mais de $80 \%$ de acerto nos 3 itens, considerado como satisfatório. Boll et al. ${ }^{3}$ em seu estudo avaliando o conhecimento prático de enfermeiros em um hospital especializado relataram que $98,8 \%$ da amostra não observaram os itens como descanso do paciente, esvaziamento da bexiga e ingestão prévia de alimentos. No mesmo estudo os itens sobre o posicionamento do braço na altura do coração, palma da mão voltada para cima, pés apoiados e o dorso do corpo relaxado atingiu o percentual de $72,9 \%$, cuidados esses preconizados pelas Diretrizes de Hipertensão para evitar interferência nos resultados.

Do total dos enfermeiros participantes da pesquisa apenas $56 \%$ acertaram o item relacionado à palpação do pulso radial para estimar o nível da pressão sistólica e $59 \%$ o item relacionado à prática de inflar 20 a $30 \mathrm{mmHg}$ a mais do nível estimado da pressão sistólica e proceder a deflação na velocidade de $2 \mathrm{mmHg} / \mathrm{s}$. Dados semelhantes foram descritos no estudo de Rabello et al. ${ }^{6}$, ao avaliarem o conhecimento prático de profissionais da área da saúde sobre a medida da pressão arterial, foi observado um número reduzido de profissionais que fizeram a palpação da artéria radial para estimação da pressão sistólica. No estudo de Veiga et al. ${ }^{9}$ foi observado que somente $53,3 \%$ dos avaliados desinsuflaram o manguito com velocidade entre 2-3 $\mathrm{mmHg} / \mathrm{s}$, pois desinsuflar o manguito com velocidade acima do recomendado, dificulta o estabelecimento preciso dos valores sistólicos e diastólicos. Para evitar falsos resultados na medida da pressão arterial, recomenda-se a palpação da artéria radial e inflação do manguito.

No desaparecimento da pulsação, o manguito deve ser desinsulflado rapidamente e aguardar pelo menos um minuto para reiniciar o procedimento. Realizando essa manobra além de estimar o valor da pressão sistólica, evita-se a hiperinsuflação que pode causar desconforto ao paciente e evita também hipoestimação da pressão sistólica na presença de hiato auscultatório, principalmente em pacientes idosos, devido à possível calcificação das artérias ${ }^{9}$.

No presente estudo $75 \%$ da amostra conseguiram definir teoricamente corretamente os sons de Korotkoff, porém uma porcentagem bem menor (22\%) acertaram que quando os batimentos persistirem até o nível zero, devemos determinar a pressão diastólica no abafamento dos sons e anotar valores da sistólica, diastólica e zero, conforme preconizado pelas VI Diretrizes Brasileiras de Hipertensão ${ }^{16}$. No estudo de Rabello et al. ${ }^{6}$ no qual foi aplicado um teste teórico a profissionais da área da saúde, menos de um terço assinalaram corretamente os sons que definem as pressões sistólica e diastólica e não souberam associar corretamente as fases I e $\mathrm{V}$ de Korotkoff, isso pode estar relacionado ao 
uso de estetoscópio que não tenha diafragma com campânula recomendado para auscultar sons graves.

Os achados deste estudo demonstraram que, dentre os entrevistados, $66 \%$ apresentaram ter conhecimento que para pacientes obesos são necessários manguitos mais longos e largos. Enquanto resultados descritos por Rabello et al. ${ }^{6}$ que mais da metade dos participantes do seu estudo apresentaram resultados insatisfatórios, destacando o conhecimento insuficiente sobre as dimensões do manguito, refletindo na prática, mesmo oferecendo diferentes manguitos e fita métrica, os observadores não mediram a circunferência do braço do paciente. Quando o manguito é muito pequeno e estreito em relação ao braço ou maior ou mais largo acarretará imprecisão dos resultados obtidos na verificação da PA, sendo necessária uma revisão nas práticas adotadas.

A recomendação é que a largura do manguito deva corresponder a $40 \%$ da circunferência do braço e o seu comprimento envolver em torno de $80 \%$, estabelecendo uma proporção de 1:2 entre a largura e o comprimento do manguito. Ainda nessa linha de pensamento, a falta de disponibilidade de diferentes tamanhos de manguitos é um sério problema enfrentado pelos profissionais da saúde, e as pesquisas sobre o assunto estão tentando atrair a atenção dos especialistas para possíveis diagnósticos errôneos de hipertensão $0^{7,12}$.

Em relação ao item sobre comunicar o valor da pressão ao paciente, um pouco mais de $83 \%$ dos participantes afirmaram que realizam essa etapa. Boll et al. $^{3}$ descreveram em seu estudo que os acertos sobre esse item deveriam ter sido atingido $100 \%$, pois a comunicação representa acolhimento e respeito ao ser humano e é essencial a qualquer cuidado prestado, ou seja, todos os pacientes deveriam ser comunicados o valor da sua pressão arterial.

\section{CONCLUSÃO}

A correta verificação da pressão arterial é indispensável na busca de dados confiáveis e do diagnóstico preciso de hipertensão arterial. Os profissionais pesquisados não estão seguindo em sua plenitude as recomendações das Diretrizes Brasileiras de Hipertensão Arterial, porém apresentou melhores resultados em vários aspectos comparados a outras pesquisas. Os resultados evidenciam deficiências em alguns conceitos teóricos, essenciais na técnica de verificação da pressão arterial, reforçando a necessidade de um aprofundamento desse assunto nos cursos de graduação, bem como treinamentos práticos aos referidos profissionais. Sendo assim será apresentada pelas pesquisadoras à gerente de enfermagem da instituição participante uma proposta para implantação de um protocolo de verificação da pressão arterial.

\section{CONFLITO DE INTERESSE}

Os autores declaram não haver qualquer potencial conflito de interesse que possa interferir na imparcialidade deste trabalho cientifico.

\section{REFERÊNCIAS}

1. Assis MMV, Oliveira JBB. Medida indireta da pressão arterial: conhecimento teórico dos fisioterapeutas. RBPS. 2003;16(1/2):17-20. DOI: http://dx.doi.org/10.5020/326

2. Cordella MP, Palota L, Cesarino CB. Medida indireta de pressão arterial: um programa de educação continuada para a equipe de enfermagem em um hospital de ensino. Arq Ciênc Saúde. 2005;12(1):21-6.

3. Boll LFC, Irigoyuen MC, Goldmeier S. Diretrizes brasileiras de hipertensão arterial: realidade da enfermagem em hospital especializado. Acta Paul Enferm. 2012;(5):775-80. DOI: http://dx.doi.org/10.1590/S0103-

\section{0}

4. Brito ES, Pantarotto RFR, Costa LRLG. A hipertensão arterial sistêmica como fator de risco ao acidente vascular encefálico (AVE). Int Health Inst. 2011;29(4):265-8.

5. Didier MT, Guimaraes AC. Otimização de recursos no cuidado primário da hipertensão arterial. Arq Bras Cardiol. 2007;88(2):218-24. http://dx.doi.org/10.1590/S0066-

\section{X2007000200014}

6. Rabello CCP, Pierim AMG, Mion Jr. D. O conhecimento de profissionais da área da saúde sobre a medida da pressão arterial. Rev Esc Enferm USP. 2004;38(2):127-34.

7. Lima LT, Gusmão JL. Conhecimento teórico e pratico de auxiliares de enfermagem sobre medida da pressão arterial. Rev Saúde. 2008;2(1):12-6

8. Geleitete TJM, Coelho EB, Nobre F. Medida casual da pressão arterial. Rev Bras Hipertens. 2009;16(2):118-122. 
9. Veiga EV, Nogueira MS, Cárnio EC, Marques S, Lavrador MAS, Moraes SA, Souza LAC, Lima NKC, Nobre F. Avaliação de técnicas da medida da pressão arterial pelos profissionais de saúde. Arq Bras Cardiol, 2003; 80(1):83-89.

10. Faertein E, Chor D, Griep RH, Alves MGM, Werneck GL, Lopes CS. Aferição da pressão arterial: experiência de treinamento de pessoal e controle de qualidade no estudo Pró-Saúde. Cad Saúde Pública. 2006;22(9):1997-2002. DOI: http://dx.doi.org/10.1590/S0102-

\section{X2006000900031}

11. Nobre F, et al. Assessment of sphygmonanometers: a proposal for excellence in blood pressure measurement. Arq. Bras. Cardiol. 2009;93(2):27-9.

DOI: http://dx.doi.org/10.1590/S0066-

\section{X2009000800026}

12. Freitas CCQ, Pantarotto RFR, Costa LRLG. Relação circunferência braquial e tamanho de manguitos utilizados nas unidades básicas de saúde de uma cidade do interior paulista. J Health Sci Inst. 2013;31(3):48-52.

13. Silva SSBE, Colosimo FC, Pierim AMG. O efeito de intervenções educativas no conhecimento da equipe de enfermagem sobre hipertensão arterial. Rev Esc Enferm USP. 2010;44(2):488-96.

14. Polit DF, Beck CT, Hungler BP. Fundamentos de pesquisa em enfermagem: métodos, avaliação e utilização. 5.ed. Porto Alegre: Artmed; 2004.

15. Severino AJ. Metodologia do trabalho científico. 24.ed. São Paulo: Cortez; 2016.

16. Sociedade Brasileira de Cardiologia / Sociedade Brasileira de Hipertensão / Sociedade Brasileira de Nefrologia. VI Diretrizes Brasileiras de Hipertensão. Arq Bras Cardiol. 2010;95(supl. 1):1-51.

Recebido para publicação em 27/03/2014

Revisado em 28/08/2016

Aceito em 14/06/2017 